\title{
12B and MGIT
}

National Cancer Institute

\section{Source}

National Cancer Institute. 12B and MGIT. NCI Thesaurus. Code C88116.

A proprietary system for culture and resistance testing of Mycobacterium samples that utilizes mycobacteria growth indicator tubes (MGIT). 